\title{
Harmonic influence analysis of unified power flow controller based on modular multilevel converter
}

\author{
Yubo YUAN ${ }^{1}$, Peng LI $^{1}$, Xiangping KONG ${ }^{1}$, Jiankun LIU ${ }^{1}$, \\ Qun LI ${ }^{1}$, Ye WANG ${ }^{1}$
}

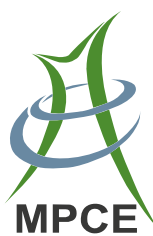

\begin{abstract}
The unified power flow controller (UPFC) based on modular multilevel converter (MMC) is the most creative flexible ac transmission system (FACTS) device. In theory, the output voltage of the series MMC in MMCUPFC can be regulated from 0 to the rated value. However, there would be relatively large harmonics in the output voltage if the voltage modulation ratio is small. In order to analyze the influence of MMC-UPFC on the harmonics of the power grid, the theoretical calculation method and spectra of the output voltage harmonics of MMC are presented. Subsequently, the calculation formulas of the harmonics in the power grid with UPFC are proposed. Based on it, the influence of UPFC on the grid voltage harmonics is evaluated, when MMC-UPFC is operated with different submodular numbers and voltage modular ratios. Eventually, the proposed analysis method is validated using digital
\end{abstract}

CrossCheck date: 13 December 2015

Received: 16 November 2015/Accepted: 17 December 2015/ Published online: 16 January 2016

(C) The Author(s) 2016. This article is published with open access at Springerlink.com

$\triangle$ Xiangping KONG

kongxphust@163.com

Yubo YUAN

yyb97104@sina.com

Peng LI

roclarry@163.com

Jiankun LIU

jiankun-liu@163.com

Qun LI

qun_li@sina.com

Ye WANG

jsnjwy_1984@163.com

1 State Grid Jiangsu Electric Power Company Research Institute, Nanjing 211103, Jiangsu, China simulation. The study results would provide guideline for the design and operation of MMC-UPFC project.

Keywords Unified power flow controller (UPFC), Modular multilevel converter (MMC), Harmonic features, Voltage modulation ratio, Submodular number

\section{Introduction}

In order to improve the performance of the existing power systems, the flexible ac transmission system (FACTS) devices have been widely applied in the power systems [1]. Among the FACTS device family, the unified power flow controller (UPFC) is the most creative FACTS device, which can simultaneously regulate multi electrical parameters to improve the power transfer capability and stability level of the power grid [2].

Since the modular multilevel converter (MMC) has the advantages of modular structure, inherent redundancy and ease of expandability, it has successfully made its way into industrial high voltage and larger power applications [3-5]. The carrier phase shifted sinusoidal pulse width modulation (CPS-SPWM) or the nearest level modulation (NLM) is usually adopted by the MMC to reduce the switching frequency, voltage and current harmonics [4-6].

The basic principle of NLM is to calculate the number of submodules (SMs) which should be put into operation based on the voltage modulation ratio, and then generate trigger pulses according to the sort of the SMs' capacitor voltages. If the voltage levels reach to a large number (such as tens or hundreds), the NLM stands out among other modulation methods, since it has the following advantages: ease of implementation, low switch frequency, and small output voltage harmonics [7, 8]. 
For the voltage source converter based high voltage direct current (VSC-HVDC) application, the single phase unit of the MMC consists of hundreds of SMs, and the output voltage of MMC is usually around the rated value under normal operation condition. In this context, the harmonics in the output voltage are very small $[4,8]$.The dc side harmonic in MMC is studied in $[9,10]$. The analytical approaches to calculate the harmonics for MMC with different modulation methods are presented in [7, 11]. A modular harmonic domain modeling technique for UPFC is developed in [12]. However, being different from the operation condition of the VSC-HVDC, the output voltage of the series MMC in MMC-UPFC can be regulated from 0 and the rated value $[13,14]$. The waveform of the output voltage would be distorted if the voltage is not so large, and the harmonics would be rather remarkable. Hence, the research on the harmonic characteristics of MMC-UPFC is of great significance to the safe and stable operation of the power grid with UPFC.

To this end, the influence of the MMC-UPFC on the harmonics of the power grid is analyzed in this paper. The structure and operating principle of MMC-UPFC is put forward. The theoretical calculation method and spectra of the output voltage harmonics of the MMC based on NLM are proposed. Then the harmonic study approach of the power grid with MMC-UPFC is presented. Finally, the simulation study which validates the proposed harmonic analysis approach is demonstrated. The harmonic suppression measures are discussed.

\section{MMC-UPFC model}

The topology of the MMC is depicted in Fig. 1 [15].

It can be found that the MMC consists of three parallelconnected phase units, each with upper and lower arms. The single arm has a modular structure with $N$ seriesconnected submodules (SMs) and a series inductance. Each SM contains two insulated-gate bipolar transistor (IGBT)/diode switches and a capacitor [4, 5].

The MMC-UPFC consists of shunt MMC and series MMC which are connected back-to-back with the common dc bus [13], as shown in Fig. 2. The shunt MMC interfaces to the power grid through the shunt transformer, and can absorb or generate controllable reactive power for AC bus voltage regulation. Meanwhile, it can exchange the active power with the power grid to maintain the dc bus voltage constant. In addition, the series MMC interfaces to the power grid through the series transformer. The power flow of the transmission line can be regulated by adjusting the magnitude and phase angle of the voltage injected by series MMC [1, 2].

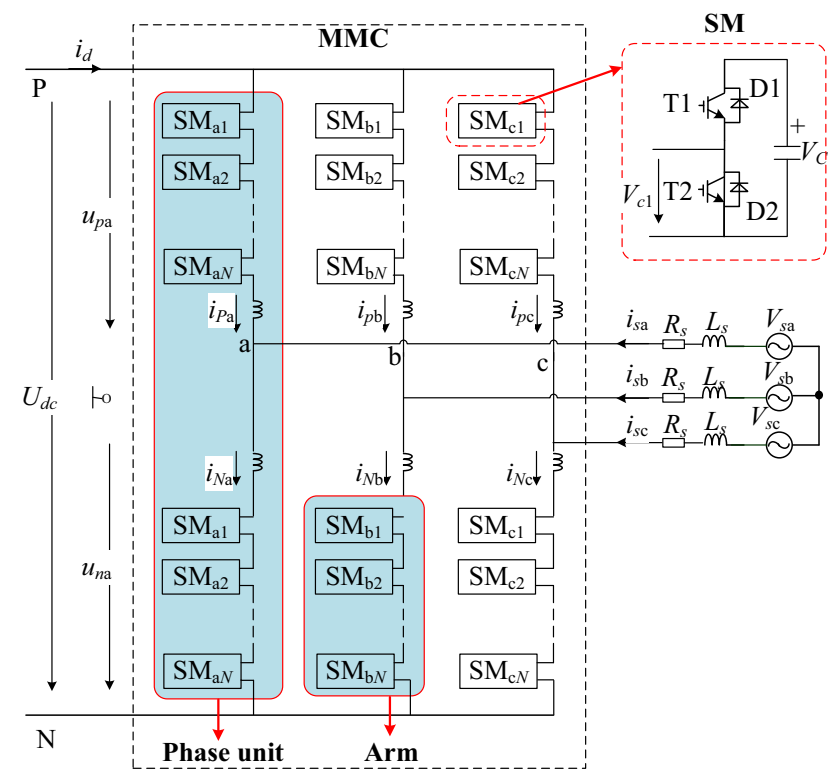

Fig. 1 The topology of MMC

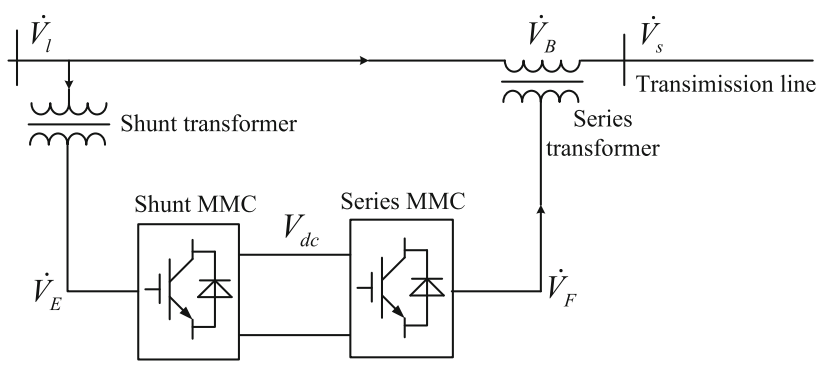

Fig. 2 System structure of UPFC

\section{Harmonic spectra of MMC based on NLM}

\subsection{Basic principle of NLM}

At present, the NLM is the most commonly used modulation technology for MMC, whose basic principle can be elaborated as follows [7]:

According to the operating principle of MMC, the number of SMs which are put into operation at every switch cycle should always be $N(N$ is number of the SMs in the single arm). $u_{r e f}$ is the modulation voltage, $V_{C}$ is the capacitor voltage of single SM.

With the increase of the instantaneous value of $u_{r e f}$, the number of SMs which are put into operation in the lower arm should increase, and the number of SMs which are put into operation in the upper arm should decrease at the same time. Consequently, the output voltage would increase. At every switch cycle, the numbers of SMs which are put into operation in the upper and lower arms can be determined according to (1). 


$$
\left\{\begin{array}{l}
n_{p}=\frac{N}{2}-\left[\frac{u_{r e f}}{V_{C}}\right], 0 \leq n_{p} \leq N \\
n_{n}=\frac{N}{2}+\left[\frac{u_{r e f}}{V_{C}}\right], 0 \leq n_{n} \leq N
\end{array}\right.
$$

where $n_{p}$ is the number of SMs which are put into operation in the upper arm; $n_{n}$ is the number of SMs which are put into operation in the lower arm; $[x]$ is the integer which is the closest to $x$.

Theoretically, the error between the output voltage and the instantaneous value of the modulation voltage can be controlled within $\pm V_{C} / 2$.

\subsection{Analysis on the output voltage harmonics of MMC}

Assume that the modulation voltage of phase $j$ (which is denoted by $u_{r e f} j$, and $j$ represents $\mathrm{a}, \mathrm{b}$ and $\mathrm{c}$ ) is the sinusoidal wave and can be expressed as

$u_{\text {ref } j}=U_{m} \sin \omega t$

where $U_{m}$ is the peak value of the modulation voltage.

According to the definition given in [2], the voltage modulation ratio $m$ can be expressed in (3).

$m=\frac{U_{m}}{\frac{1}{2} U_{d c}}$

where $U_{d c}$ is the dc bus voltage.

Assume each SM has the same capacitor voltage, then

$U_{d c}=N V_{c}$

Hence, the following expression can be obtained.

$u_{r e f j}=\frac{m N}{2} V_{C} \sin \omega t$

Then the numbers of SMs which are put into operation in the upper and lower arms of the $j$ phase unit are

$\left\{\begin{array}{l}n_{p j}=\frac{N}{2}-\left[\frac{m N}{2} \sin \omega t\right], 0 \leq n_{p j} \leq N \\ n_{n j}=\frac{N}{2}+\left[\frac{m N}{2} \sin \omega t\right], 0 \leq n_{n j} \leq N\end{array}\right.$

Hence, the output voltage of phase $j$ is

$u_{v j}=\frac{u_{n j}-u_{p j}}{2}=\left[\frac{m N}{2} \sin \omega t\right] V_{C}=K V_{C}$

where $u_{p j}$ and $u_{n j}$ are respectively the upper arm voltage and lower arm voltage of the phase $j$ unit; $K$ is the integer and satisfies the following condition:

$K-\frac{1}{2}<\frac{m N}{2} \sin \omega t \leq K+\frac{1}{2}$

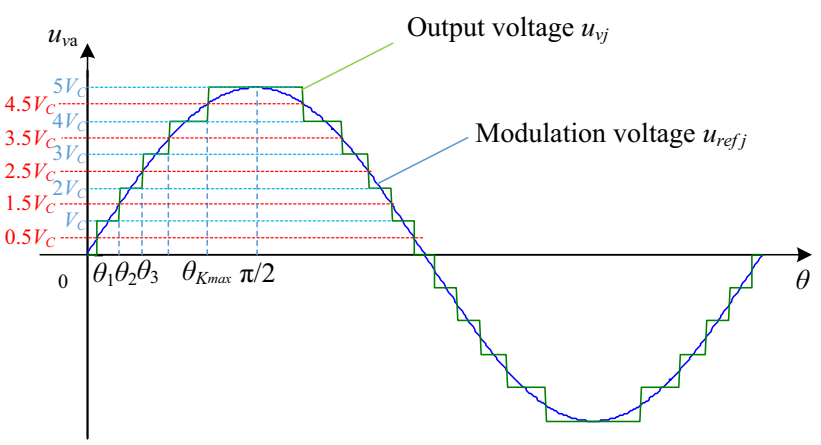

Fig. 3 Modulation voltage and output voltage of MMC

Taking the MMC with 11 voltage levels for example, Fig. 3 depicts the modulation voltage and the output voltage of MMC.

In Fig. 3, $K_{\max }$ is the number of the voltage levels in the first quarter period (for the MMC with 11 voltage levels, $K_{\max }$ should be 5 ). The electrical angles of each voltage level are respectively denoted by $\theta_{1}, \ldots, \theta_{K}, \ldots, \theta_{\text {Kmax }}$.

According to (8), (9) can be obtained.

$-\frac{m N}{2}-\frac{1}{2} \leq K<\frac{m N}{2}+\frac{1}{2}$

Meanwhile, $K$ should satisfy the following condition:

$-\frac{N}{2} \leq K \leq \frac{N}{2}$

Since it is an integer, $K_{\max }$ should be

$K_{\text {max }}=\min \left(\left\lfloor\frac{m \cdot N}{2}+\frac{1}{2}\right\rfloor, \frac{N}{2}\right)$

where $\lfloor x\rfloor$ is the largest integer that smaller than $x$, and min $(a, b)$ is to obtain the smaller one of $a$ and $b$.

Besides, $\theta_{K}$ should satisfy the following expression.

$K-\frac{1}{2}=\frac{m N}{2} \sin \left(\theta_{K}\right), \quad K=1,2, \cdots, K_{\max }$

Hence,

$\theta_{K}=\arcsin \left(\frac{2 K-1}{m \cdot N}\right), \quad K=1,2, \cdots, K_{\text {max }}$

Moreover, apply Fourier decomposition to the output voltage of MMC, and (14) can be obtained.

$u_{v j}=a_{0 j}+\sum_{s=1,2, \cdots}\left[a_{s j} \cos (s \omega t)+b_{s j} \sin (s \omega t)\right]$

As Fig. 3 shows, output voltage of MMC is odd symmetry, subsequently,

$a_{s}=0, \quad s=0,1,2, \cdots$

$b_{s}=0, \quad s=2,4,6 \cdots$ 


$$
\begin{aligned}
b_{s}= & \frac{8}{T} \int_{0}^{\frac{T}{4}} u_{v j} \sin (s \omega t) \mathrm{d} t= \\
& \frac{8}{\omega T} \sum_{K=1}^{K_{\max }} \int_{\theta_{K}}^{\theta_{K+1}} K V_{c} \sin (s \theta) \mathrm{d} \theta, s=1,3,5, \cdots= \\
& \frac{4 V_{c}}{\pi} \frac{1}{s} \sum_{K=1}^{K_{\max }} \cos \left(s \theta_{K}\right)
\end{aligned}
$$

where $\theta_{K_{\max }+1}=\pi / 2$.

Hence, the output voltage of MMC can be expressed by

$u_{v j}=\frac{4 V_{c}}{\pi} \sum_{s=1,3, \cdots}\left[\frac{1}{s} \sum_{K=1}^{K_{\max }} \cos \left(s \theta_{K}\right)\right] \cdot \sin (s \omega t)$

It can be found that there are odd harmonics, but no even harmonic in the output voltage of MMC. Moreover, the content of each harmonic can be calculated with (19).

$H u_{v j}(s)=\frac{\frac{1}{s} \sum_{K=1}^{K_{\max }} \cos \left(s \theta_{K}\right)}{\sum_{K=1}^{K_{\max }} \cos \left(\theta_{K}\right)} \quad s=3,5,7, \cdots$

\section{Harmonic analysis of UPFC}

Since the output voltage of the shunt MMC in UPFC is always close to the rated value, and the voltage modulation ratio is close to 1.0, the harmonics in the output voltage of the shunt MMC and their influence on the power grid are very small. Hence, the harmonics in the output voltage of the shunt MMC is not discussed here.

In order to study the influence of the series MMC in UPFC on the harmonics of the power grid, Fig. 4 gives the equivalent circuit of the power grid with UPFC.

In Fig. 4, the series part of UPFC (consists of series $\mathrm{MMC}$ and series transformer) is entirely represented by the equivalent voltage source $e_{v}$ which is series-connected in the transmission line $M N$ at Bus $M$. The equivalent system sources at the two terminals are represented by $e_{m}$ and $e_{n}$, the equivalent inductances of the two voltage sources are respectively denoted by $L_{m}$ and $L_{n}$. Besides, the resistance of the transmission line $M N$ is ignored since it is very small, and the inductance is $L_{l}$.

It can be obtained from Fig. 4 that

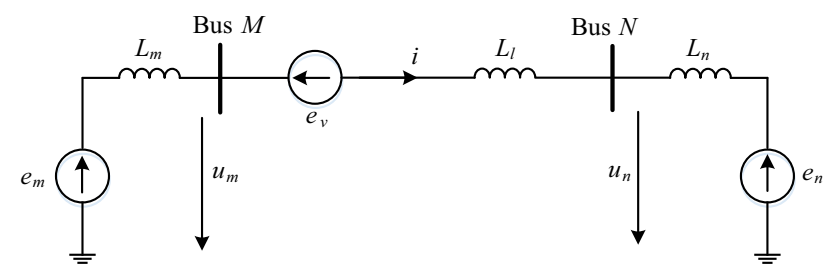

Fig. 4 The equivalent circuit of the power grid with UPFC

$$
\begin{gathered}
e_{m}=e_{v}+e_{n}+\frac{\left(L_{m}+L_{l}+L_{n}\right) \mathrm{d} i}{\mathrm{~d} t} \\
\left\{\begin{array}{l}
u_{n}=e_{n}+L_{n} \frac{\mathrm{d} i}{\mathrm{~d} t} \\
u_{m}=e_{m}-L_{m} \frac{\mathrm{d} i}{\mathrm{~d} t}
\end{array}\right.
\end{gathered}
$$

Accordingly, the voltages of Bus $M$ and Bus $N$ which are denoted by $u_{m}$ and $u_{n}$ are respectively:

$\left\{\begin{array}{l}u_{n}=\frac{e_{m} L_{n}+e_{n}\left(L_{m}+L_{l}\right)}{L_{m}+L_{l}+L_{n}}-\frac{L_{n}}{L_{m}+L_{l}+L_{n}} e_{v} \\ u_{m}=\frac{e_{m}\left(L_{l}+L_{n}\right)+e_{n} L_{m}}{L_{m}+L_{l}+L_{n}}+\frac{L_{m}}{L_{m}+L_{l}+L_{n}} e_{v}\end{array}\right.$

Moreover, the equivalent circuit of the series transformer in UPFC is given in Fig. 5.

In Fig. 5, $u_{v}$ is the output voltage of the series MMC, $e_{p}$ is the voltage of the series transformer's primary winding. $e_{p}=k u_{v}$, and $k$ is the turns ratio of the series transformer. $L_{T}$ and $L_{T m}$ are respectively the equivalent leakage inductance and magnetic inductance of the series transformer.

Hence, the detailed equivalent circuit of the power grid with UPFC is depicted in Fig. 6.

By replacing $L_{l}$ and $e_{v}$ in (22) respectively with $L_{l}+L_{T}$ and $k u_{v j}$, the following expression can be obtained.

$$
\left\{\begin{array}{l}
u_{n}=\frac{e_{m} L_{n}+e_{n}\left(L_{m}+L_{l}+L_{T}\right)}{L_{m}+L_{l}+L_{T}+L_{n}}-\frac{L_{n}}{L_{m}+L_{l}+L_{T}+L_{n}} k \cdot u_{v j} \\
u_{m}=\frac{e_{m}\left(L_{l}+L_{T}+L_{n}\right)+e_{n} L_{m}}{L_{m}+L_{l}+L_{T}+L_{n}}+\frac{L_{m}}{L_{m}+L_{l}+L_{T}+L_{n}} k \cdot u_{v j}
\end{array}\right.
$$

It can be found that $u_{m}$ and $u_{n}$ both consist of two parts. The first parts are fundamental frequency components and only related to the equivalent system sources. The second parts are related to the output voltage of the series MMC. Hence, there are also odd harmonics in $u_{m}$ and $u_{n}$.

Rewrite (23) in the following forms:

$$
\left\{\begin{array}{l}
u_{n}=A_{n} \sin \left(\omega t+\varphi_{n}\right)+B_{n} \cdot u_{v j} \\
u_{m}=A_{m} \sin \left(\omega t+\varphi_{m}\right)+B_{m} \cdot u_{v j}
\end{array}\right.
$$

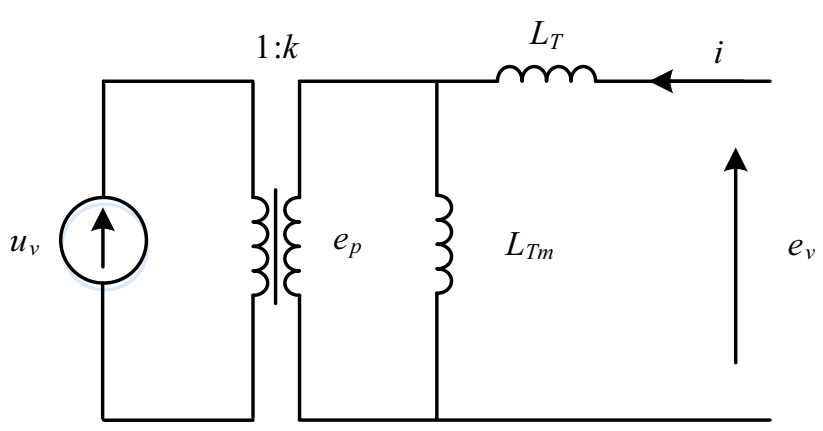

Fig. 5 Equivalent circuit of the series transformer 


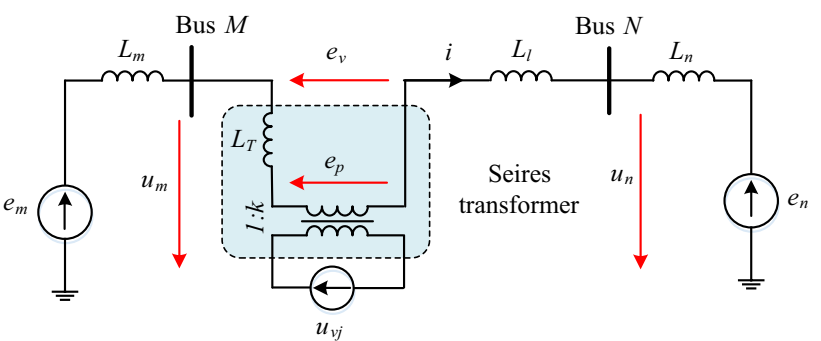

Fig. 6 The detailed equivalent circuit of the power grid with UPFC

where $A_{n}, \varphi_{n}, A_{m}$ and $\varphi_{m}$ can be determined by applying triangular calculation to the first parts of $u_{m}$ and $u_{n}$. $B_{n}$ and $B_{m}$ satisfy the following conditions.

$\left\{\begin{array}{l}B_{n}=-\frac{L_{n} k}{L_{m}+L_{l}+L_{T}+L_{n}} \\ B_{m}=\frac{L_{m} k}{L_{m}+L_{l}+L_{T}+L_{n}}\end{array}\right.$

According to (17), (18) and (24), the harmonic contents in $u_{m}$ and $u_{n}$ can be calculated with (26).

$$
\begin{aligned}
& \left\{\begin{array}{c}
H u_{n}(s)=\frac{B_{n} b_{s}}{\sqrt{A_{n}^{2}+2 A_{n} b_{1} B_{n} \cos \varphi_{n}+\left(b_{1} B_{n}\right)^{2}}} \\
H u_{m}(s)=\frac{B_{m} b_{s}}{\sqrt{A_{m}^{2}+2 A_{m} b_{1} B_{m} \cos \varphi_{m}+\left(b_{1} B_{m}\right)^{2}}}
\end{array}\right. \\
& s=3,5,7, \cdots
\end{aligned}
$$

\section{Simulation analysis}

\subsection{Output voltage harmonics of MMC}

For different voltage modulation ratios, Fig. 7 demonstrates the harmonic contents in the output voltage of MMC, whose orders are not larger than 51. It is noted that $N=26, U_{d c}=40 \mathrm{kV}$.

Besides, for different SM numbers, the relationship between the total harmonic distortion (THD) in the output voltage of MMC and the voltage modulation ratio $m$ is given in Fig. 8.

According to the results shown in Fig. 7 and Fig. 8, the following conclusions can be drawn.

1) There are odd harmonics in the output voltage of MMC.

2) If $m \leq 1.0$, with the increase of $m$, the harmonic contents would decrease. When $m$ reaches to 1.0 , the harmonic contents are the smallest. If $m>1.0$, the harmonic contents would increase greatly.

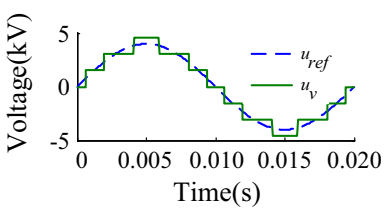

(a) Output voltage of MMC with $m=0.2$

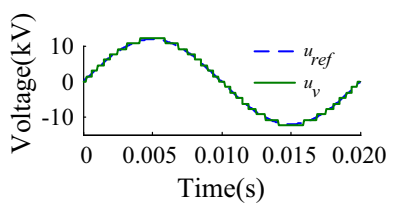

(c) Output voltage of MMC with $m=0.6$

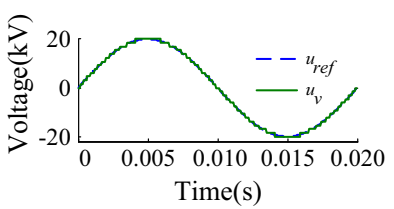

(e) Output voltage of MMC with $m=1.0$

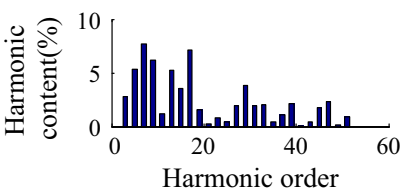

(b) Harmonic spectra with $m=0.2$

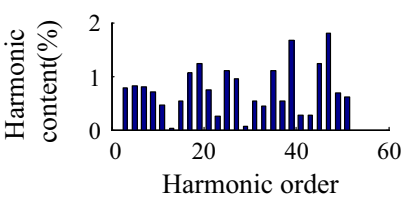
$m=0.6$

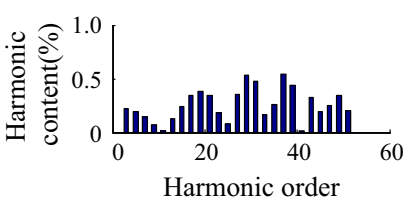

(f) Harmonic spectra with $m=1.0$ (d) Harmonic spectra with

Fig. 7 Output voltages of MMC and the harmonic contents

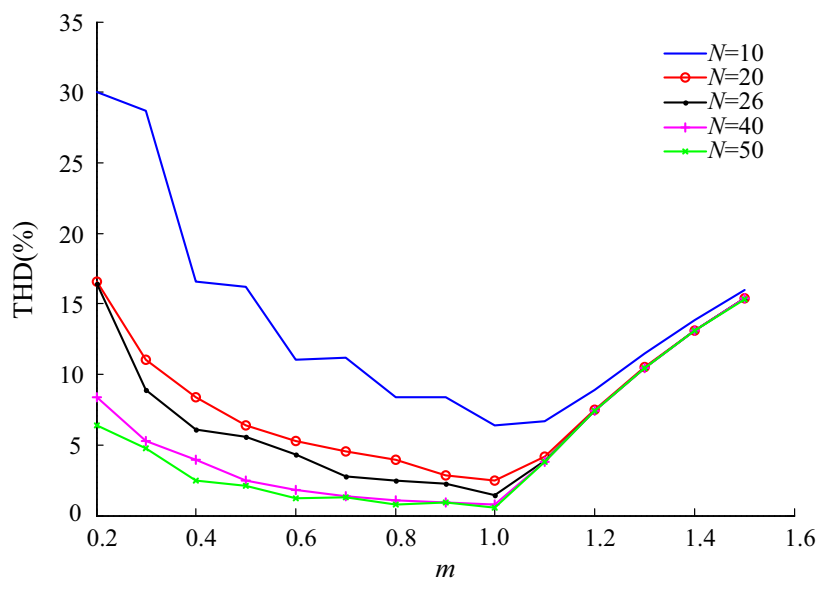

Fig. 8 Relationship between THD of output voltage and modulate ratio

3) With the increase of $N$, the harmonic contents in the output voltage of MMC would decrease. However, if $N$ is larger than 20, with the increase of $N$, the improvement of the harmonic contents would be not that significant.

\subsection{Harmonic analysis of the power grid with UPFC}

The parameters of the components in Fig. 6 are: $L_{m}=0.1 \mathrm{H}, L_{n}=0.2 \mathrm{H}, L_{l}=0.02 \mathrm{H}, L_{T}=0.5 \mathrm{H}$, $k=26.5 / 20.8 \mathrm{kV}, e_{m}=127 \sin (\omega t+0.175) \mathrm{kV}, e_{n}=127-$ $\sin \omega t \mathrm{kV}$. 


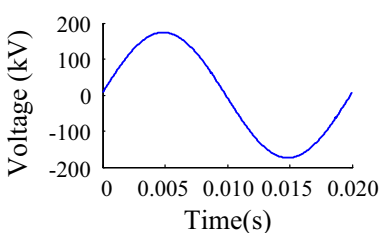

(a) Voltage of Bus $N$ with $m=0.8$

(c) Voltage of Bus $M$ with $m=0.8$

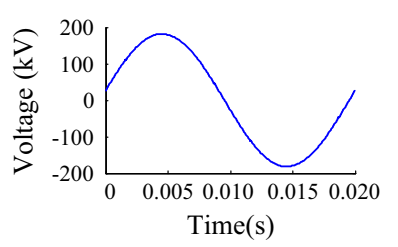

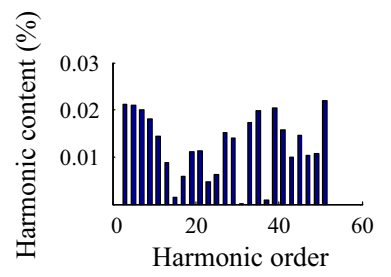

(b) Harmonic spectra with

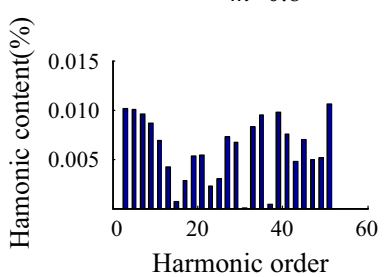

(d) Harmonic spectra with
Fig. 9 Bus voltages and harmonic contents $(m=0.8)$

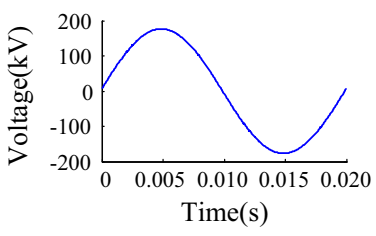

(a) Voltage of Bus $N$ with $m=0.4$

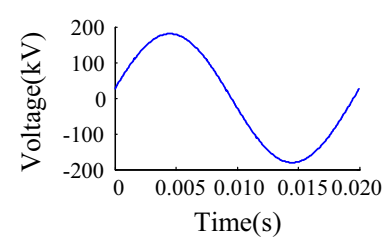

(c) Voltage of Bus $M$ with $m=0.4$

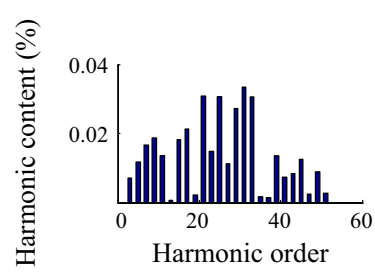

(b) Harmonic spectra with $m=0.4$

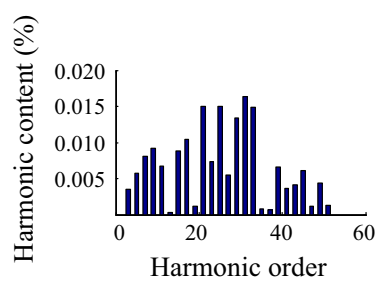

(d) Harmonic spectra with $m=0.4$
Fig. 10 Bus voltages and harmonic contents $(m=0.4)$

For $N=26$ and $U_{d c}=40 \mathrm{kV}$, the waveforms of $u_{m}$ and $u_{n}$, as well as their harmonic contents with different voltage modulation ratios are depicted in Fig. 9 and Fig. 10. Besides, for different SM numbers, the relationships between the THD of $u_{m}, u_{n}$ and the voltage modulation ratio $m$ are given in Fig. 11 .

Based on the results shown in Fig. 9, Fig. 10 and Fig. 11, the following conclusions can be drawn.

1) There are odd harmonics in the bus voltages, related to the odd harmonics in the output voltage of MMC.

2) If $m<1.0$, with the increase of $m$, the harmonic contents decreases slightly. When $m$ reaches to 1.0 , the

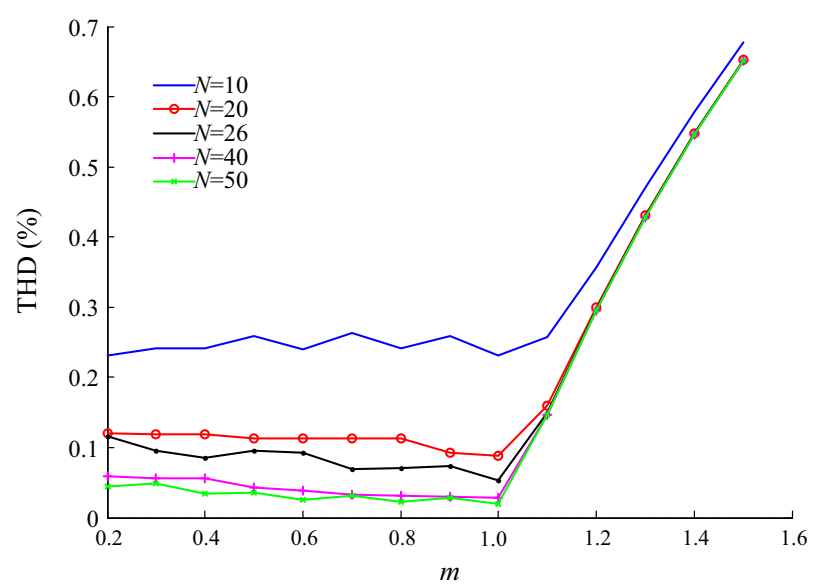

(a) THD of voltage at Bus $N$

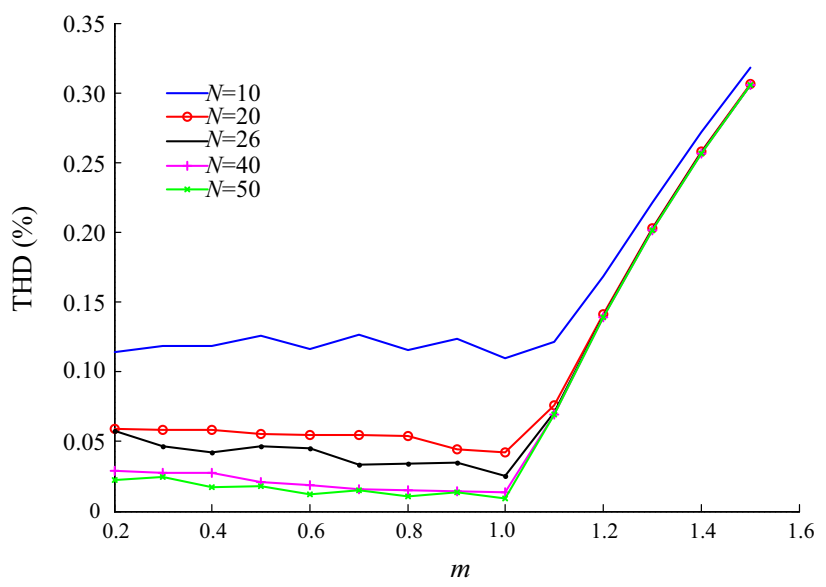

(b) THD of voltage at Bus $M$

Fig. 11 Relationships between THD of bus voltages and modulate ratio

harmonic contents are the smallest. If $m>1.0$, the harmonic contents would increase greatly with the increase of $m$.

3) With the increase of $N$, the harmonic contents in the bus voltages would decrease. However, if $N$ is larger than 20, with the increase of $N$, the improvement of the harmonic contents would be not that significant.

\subsection{Simulation results}

The simulation model of the power grid with UPFC is built using PSCAD/EMTDC, where $N=26, U_{d c}=40 \mathrm{kV}$, and NLM is adopted. Besides, the parameters of the power grid are configured according to a practical UPFC project.

With different voltage modulation ratios, Fig. 12 shows the comparison of the theoretical results and simulation results of the series MMC's output voltage, and Fig. 13 demonstrates the comparison of the theoretical results and simulation results of the system voltage. 


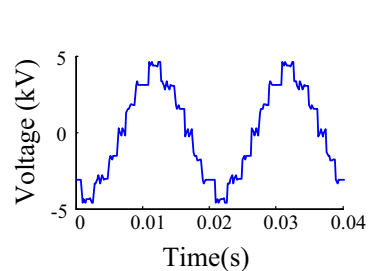

(a) Output voltage of MMC with

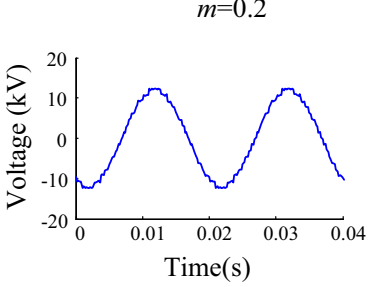

(c) Output voltage of MMC with $m=0.6$

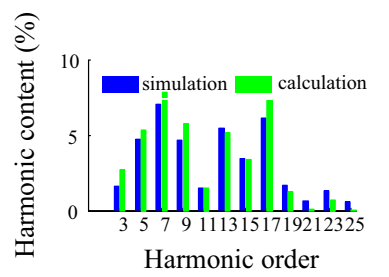

(b) Harmonic spectra with

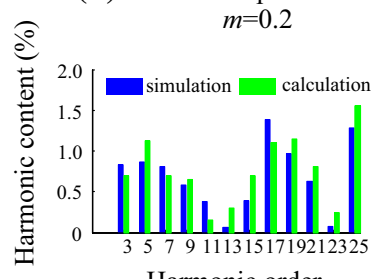

Harmonic order

(d) Harmonic spectra with $m=0.6$

Fig. 12 Output voltages of series MMC and the harmonic contents

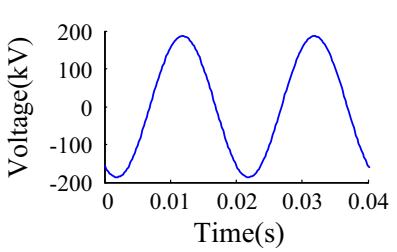

(a) System voltage with $m=0.2$

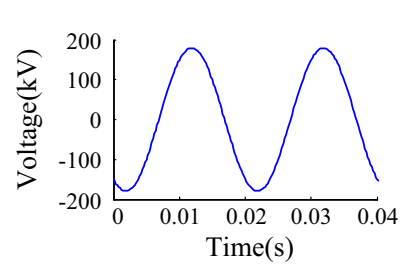

(c) System voltage with $m=0.6$

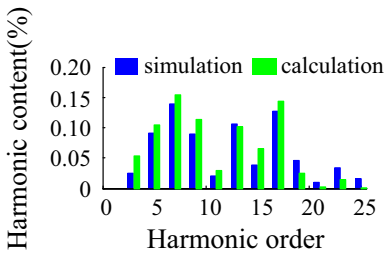

(b) Harmonic spectra with $m=0.2$

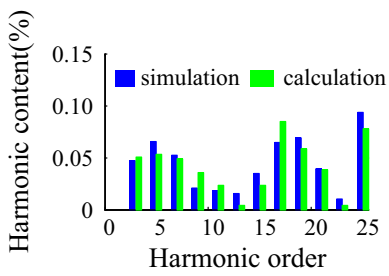

(d) Harmonic spectra with $m=0.6$

Fig. 13 System voltage and harmonic contents

It can be found that the simulation results about the harmonic contents of the series MMC's output voltage and the system voltage agree with the theoretical results rather well, which validate the effectiveness of the proposed theoretical analysis method.

\section{Influence factors of harmonics in the power grid and countermeasures}

\subsection{Influence analysis of UPFC on harmonics}

It can be found from the above analysis that the harmonics of the power grid caused by MMC-UPFC are

Table 1 Maximum harmonic content and THD under different AC system voltages

\begin{tabular}{lll}
\hline $\begin{array}{l}\text { AC system voltage } \\
(\mathrm{kV})\end{array}$ & $\begin{array}{l}\text { Maximum harmonic content } \\
(\%)\end{array}$ & $\begin{array}{l}\text { THD } \\
(\%)\end{array}$ \\
\hline 10 & 1.73 & 3.98 \\
35 & 0.34 & 0.79 \\
110 & 0.10 & 0.23 \\
220 & 0.05 & 0.11 \\
500 & 0.02 & 0.05 \\
\hline
\end{tabular}

Table 2 Maximum harmonic content and THD under different rated dc bus voltages

\begin{tabular}{lll}
\hline $\begin{array}{l}\text { Rated dc bus voltage } \\
(\mathrm{kV})\end{array}$ & $\begin{array}{l}\text { Maximum harmonic content } \\
(\%)\end{array}$ & $\begin{array}{l}\text { THD } \\
(\%)\end{array}$ \\
\hline 40 & 0.049 & 0.114 \\
80 & 0.101 & 0.231 \\
100 & 0.127 & 0.291 \\
150 & 0.194 & 0.447 \\
200 & 0.265 & 0.611 \\
\hline
\end{tabular}

Table 3 Maximum harmonic content and THD with different $L_{n}$

\begin{tabular}{lll}
\hline$L_{n}(\mathrm{H})$ & Maximum harmonic content $(\%)$ & THD $(\%)$ \\
\hline 0.1 & 0.028 & 0.064 \\
0.2 & 0.049 & 0.114 \\
0.5 & 0.092 & 0.211 \\
1.0 & 0.128 & 0.298 \\
1.5 & 0.148 & 0.342 \\
\hline
\end{tabular}

related to the SM number, voltage modulation ratio, rated dc bus voltage of UPFC, and the AC system parameters.

1) Influence of the $\mathrm{AC}$ system voltage

According to (23), the smaller the voltage of the AC system is, the larger the influence of UPFC on the harmonics of the power grid is. In order to validate the theoretical analysis result, Table 1 shows the relationship between the AC system voltage and the maximum harmonic content, THD in the bus voltage of the power grid. It is noted that $m=0.5, U_{d c}=40 \mathrm{kV}$, and $N=20$.

2) Influence of rated dc bus voltage of UPFC

For the same SM number, with the increase of the rated $\mathrm{dc}$ bus voltage of UPFC, the single SM's capacitor voltage would also increase. Hence, the number of the output voltage levels would decrease when the voltage modulation ratio is small. In this context, the influence of UPFC on the bus voltage harmonics of the power grid would be larger with the 
increase of the rated dc bus voltage of UPFC. Table 2 shows the relationship between the rated dc bus voltage of UPFC and the maximum harmonic content, THD in the bus voltage of the power grid. It is noted that $m=0.5, U_{a c}=220 \mathrm{kV}$, and $N=20$.

3) Influence of $\mathrm{AC}$ system parameters

As shown in (23), the influence of UPFC on the harmonics of the power grid would increase with the decrease of the short circuit capability of the AC system. In order to validate the theoretical analysis result, Table 3 shows the relationship between $L_{n}$ and the maximum harmonic content, THD in the bus voltage of the power grid. Noted that $m=0.5$, $U_{a c}=220 \mathrm{kV}, U_{d c}=40 \mathrm{kV}$ and $N=20$.

From the above theoretical analysis and simulation study, it can be found that if the AC system voltage is rather high, its influence on the harmonics in the bus voltage of the power grid is rather small and can be ignored. If the rated dc bus voltage of the UPFC is large, or the short circuit capability of the AC system is weak, the influence of UPFC on the bus voltage harmonics of the power grid is relatively larger, which should be taken into consideration for the UPFC project design and operation. Meanwhile, special countermeasures should be taken to improve the power quality of the power grid.

\subsection{Countermeasures}

In order to reduce the influence of the MMC-UPFC on the harmonics of the power grid, some special countermeasures are presented, such as optimizing the planning and design of the UPFC project, as well as improving the control scheme of UPFC.

1) During the planning and design stage of the UPFC project, the access point of UPFC, AC system voltage and UPFC parameters (such as rated dc bus voltage and SM number) should be chosen reasonably. Moreover, the filters should be installed when necessary, though the investment would increase.

2) If the voltage modulation ratio is small, the number of the output voltage levels would decrease, which increase the harmonic contents. In order to address the above issue, improved control scheme of UPFC should be adopted. For example, the coordinate control of the series MMC and shunt MMC should be implemented, to improve the number of output voltage levels by reducing the single SM's capacitor voltage. Moreover, the margin of the voltage modulation ratio, redundant SMs and on-load tap regulation switch of the shunt transformer should be employed to increase the number of the output voltage levels. The
Table 4 The level numbers with different AC system voltage

\begin{tabular}{lll}
\hline $\begin{array}{l}\text { Peak value of } \\
\text { AC system } \\
\text { voltage }(\mathrm{kV})\end{array}$ & $\begin{array}{l}\text { Level number without } \\
\text { reducing single SM's } \\
\text { capacitor voltage }\end{array}$ & $\begin{array}{l}\text { Level number with } \\
\text { reducing single SM's } \\
\text { capacitor voltage }\end{array}$ \\
\hline 1.0 & 3 & 3 \\
1.6 & 3 & 5 \\
2.5 & 5 & 7 \\
3.7 & 7 & 9 \\
4.5 & 7 & 11 \\
\hline
\end{tabular}

above improved control scheme of UPFC would not increase the investment, and can improve the control and operation feasibility of UPFC.

The advantage of increasing number of output voltage levels on the harmonic suppression is analyzed as follows.

If $N=30, U_{d c}=40 \mathrm{kV}$, then the single SM's capacitor voltage $U_{s m}$ should be $1.33 \mathrm{kV}$. Assuming $m=0.8$, the peak value of the output voltage of the shunt MMC is

$U_{a c P}=m \frac{U_{d c}}{2}=20 \times 0.8=16 \mathrm{kV}$

On condition that the output voltage of the shunt MMC keeps constant, the following expressions can be obtained.

$\left\{\begin{array}{l}U_{d c}=2 \frac{U_{a c} P}{m}=2 U_{a c P}=32 \mathrm{kV} \\ U_{s m}=\frac{2 U_{a c P}}{N_{e}}=1.067 \mathrm{kV}\end{array}\right.$

It can be found that $U_{s m}$ reduces to be $1.067 \mathrm{kV}$.

Furthermore, if the redundant SMs whose number is 5 are put into operation, $U_{s m}$ would reduce to be $0.914 \mathrm{kV}$, as given in (29):

$U_{s m}=\frac{2 U_{a c P}}{N_{e}+N_{r e d}}=0.914 \mathrm{kV}$

where $N_{\text {red }}$ is the number of the redundant SMs.

For the different $\mathrm{AC}$ system voltages, the numbers of the output voltage levels with and without reducing single SM's capacitor voltage are given in Table 4.

It can be found that with reducing single SM's capacitor voltage, the number of the output voltage levels can be increased to reduce the harmonics injected by UPFC.

\section{Conclusion}

According to the operation of MMC-UPFC, the harmonics of the MMC-UPFC is studied in this paper. Based on the harmonic features of MMC and equivalent circuit of the power grid with UPFC, the harmonic spectra analysis 
method of the power grid is studied. Moreover, the influence of SM number, voltage modulation ratio, and $\mathrm{AC}$ system voltage on the harmonics of the power grid are analyzed. The effectiveness of the proposed analysis method is validated using PSCAD/EMTDC simulation. Finally, the countermeasures which can help to suppress the harmonics in the power grid are presented. The study results of this paper can provide guideline for the design and operation of the UPFC project.

Acknowledgment This work was supported by State Grid Corporation of China (SGCC)'s Major Science and Technology Demonstrative Project of UPFC in West Nanjing Power Grid (No. SGCC2015-011)

Open Access This article is distributed under the terms of the Creative Commons Attribution 4.0 International License (http:// creativecommons.org/licenses/by/4.0/), which permits unrestricted use, distribution, and reproduction in any medium, provided you give appropriate credit to the original author(s) and the source, provide a link to the Creative Commons license, and indicate if changes were made.

\section{References}

[1] Li CB, Xiao LW, Cao YJ et al (2014) Optimal allocation of multi-type FACTS devices in power systems based on power flow entropy. J Mod Power Syst Clean Energy 2(2):173-180. doi:10.1007/s40565-014-0059-x

[2] Gyugyi L, Schauder CD, Williams SL et al (1995) The unified power flow controller: a new approach to power transmission control. IEEE Trans Power Deliv 10(2):1085-1097

[3] Dorn J, Huang H, Retzmann D (2007) Novel voltage-sourced converters for HVDC and FACTS application. In: Proceedings of the CIGRE symposium on system development and asset management under restructuring, Osaka, Japan, 1-4 Nov 2007, 8 $\mathrm{pp}$

[4] Song JY, Feng XQ, Cui FB et al (2014) Dynamic modeling and internal characteristic simulation research of MMC-UPFC. Proc CSEE 34:67-75 (in Chinese)

[5] Tu QR, Xu Z, Zheng X et al (2010) Mechanism analysis on the circulating current in modular multilevel converter based HVDC. High Volt Eng 36(2):547-552 (in Chinese)

[6] Wang SS, Zhou XX, Tang GF et al (2011) Modeling of modular multi-level voltage source converter. Proc CSEE 31(24):1-8 (in Chinese)

[7] Tu QR, Xu Z (2011) Impact of sampling frequency on harmonic distortion for modular multilevel converter. IEEE Trans Power Deliv 26(1):298-306

[8] Guan Y, Xu Z, Tu QR et al (2010) Nearest level modulation for modular multilevel converters in HVDC transmission. Autom Electr Power Syst 34(2):48-52 (in Chinese)

[9] Wang F, Xu Z, Huang Y et al (2010) DC harmonic current calculation for HVDC systems based on the classical transmission line model. In: Proceedings of the 2010 international conference on power system technology, Hangzhou, China, 24-28 Oct 2010, 5 pp
[10] Zhang ZR, Xu Z, Xue YL et al (2015) DC-side harmonic currents calculation and DC-loop resonance analysis for an LCCMMC hybrid HVDC transmission system. IEEE Trans Power Deliv 30(2):642-651

[11] Wang LQ, Lin P, Li JL et al (2004) Harmonic characteristic of sample time staggered space vector modulation for multi-modular or multilevel converters. In: Proceedings of the IEEE 35th annual power electronics specialists conference, vol 6, Aachen, Germany, 20-25 Jun 2004, pp 4554-4557

[12] Collins C, Watson N, Wood A (2006) UPFC modeling in the harmonic domain. IEEE Trans Power Deliv 21(2):933-938

[13] Zhang ZH, Jiang DZ (2012) Control strategy simulation of UPFC based on modular multilevel converters. Power Syst Prot Control 40(3):73-77 (in Chinese)

[14] Jing P, Zhou F, Song JY et al (2013) Design and simulation of unified power flow controller based on modular multilevel converter. Power Syst Technol 37(2):356-361 (in Chinese)

[15] Wang PY, Zhang XP, Coventry PF et al (2014) Control and protection strategy for MMC MTDC system under converterside AC fault during converter blocking failure. J Mod Power Syst Clean Energy 2(3):272-281. doi:10.1007/s40565-0140064-0

Yubo YUAN received the Ph.D. degree in School of Electrical Engineering from Southeast University, Nanjing, China, in 2004. Currently, he is a professorate engineer in State Grid Jiangsu Electric Power Company Research Institute. His research interest is protective relaying.

Peng LI received the Ph.D. degree in School of Electrical Engineering from Xi' an Jiaotong University, Xi'an, China, in 2011. Currently, he is a senior engineer in State Grid Jiangsu Electric Power Company Research Institute. His research interest is control and protection of HVDC.

Xiangping KONG received the Ph.D. degree in School of Electrical and Electronic Engineering from Huazhong University of Science and Technology, Wuhan, China, in 2014. Currently, he is an engineer in State Grid Jiangsu Electric Power Company Research Institute. His research interest is protective relaying.

Jiankun LIU received the Master Degree in School ofElectrical Engineering from Xi'an Jiaotong University,Xi'an, China, in 2004. Currently, he is a Senior Engineer inState Grid Jiangsu Electric Power Company ResearchInstitute. His research interest is power system analysis.

Qun LI received the Ph.D. degree in School of Electrical Engineering from Southeast University, Nanjing, China, in 1999. Currently, he is a professorate senior engineer in State Grid Jiangsu Electric Power Company Research Institute. His research interest is power quality analysis and renewable power integration.

Ye WANG received the Ph.D. degree in School of Electrical Engineering from Southeast University, Nanjing, China, in 2013. Currently, he is an engineer in State Grid Jiangsu Electric Power Company Research Institute. His research interest is control and protection of HVDC. 